\title{
VIBRATION OF CIRCULAR PLATES SUBJECTED TO CONSTANT RADIAL LOAD IN THEIR PLANE
}

\author{
NÓRA SzÜCS \\ Robert Bosch Energy and Body Systems Kft., \\ H-3526 Miskolc, Robert Bosch Park 3., Hungary \\ noraszucs@gmail.com \\ GYÖRGY SZEIDL \\ Institute of Applied Mechanics, University of Miskolc \\ H-3515 Miskolc-Egyetemváros, Hungary \\ gyorgy.szeidl@gmail.com
}

[Received: October 18, 2016; Accepted: December 12, 2016]

\begin{abstract}
The present paper deals with the vibration of circular plates provided that the plates are prestressed in such a way that the stresses due to the in-plane load are constants. We have determined the Green functions of the governing equations. The self adjoint eigenvalue problems giving the natural frequencies are replaced by homogenous Fredholm integral equations for which the symmetric Green functions constitute the kernel. According to the numerical solution the square of the natural frequencies is linear, or approximately linear functions of the constant in plane load.
\end{abstract}

Mathematical Subject Classification: 74H45, 74H55, 74K20

Keywords: Circular plates, Green functions, natural frequencies, critical load

\section{INTRODUCTION}

It is well known [1] that the natural frequency of a simply supported beam subjected to a compressive axial force - the beam is in a prestressed state - satisfies the equation

$$
\frac{\alpha^{2}}{\alpha_{1}^{2}}=1-\frac{f}{f_{1}}
$$

in which $\alpha$ and $\alpha_{1}$ are the first natural frequencies of the loaded and unloaded beam while $f$ and $f_{1}$ are the compressive force and its smallest critical value.

Lawther [1] attacks the problem how a prestressed state of the body affects the natural frequencies in a more general form. He studies finite dimensional multiparameter eigenvalue problems and comes to the conclusion that for multiparameter problems, the eigenvalue part of the solution is described by interaction curves in an eigenvalue space, and every such eigenvalue solution has an associated eigenvector. If all points 
on a curve have the same eigenvector then the curve is necessarily a straight line, but the converse is far more complex.

Boundary element solutions for the plate buckling problem have been published among others in papers 2, 3, 4, 5, 6. The authors of the papers cited investigate the buckling phenomenon under various assumptions and use different mechanical models. However, it is a common feature of each paper that the fundamental solutions utilized do not involve the effect of the in-plane stresses directly. The reason for attacking the problem in this way is simple: the model is applicable for any in-plane load exerted on the boundary. The price one has to pay for the generality of the model concerning the in-plane load is the presence of a domain integral which should be handled in some way. However, there are various cases when the stress state due to the in-plane load is a constant one. We also remark that the papers cited above are not concerned with the issue of how the in-plane stresses affect the vibrations of the plate.

Paper [7] is devoted to the issue what vibration characteristics of centrally clamped, variable thickness disks have if they are subjected to rotational and thermal in-plane stresses. This paper contains useful references for the most important earlier results which are not cited here. Pardoen [8] turned his attention to the vibration of prestressed circular orthotropic plates and used a finite element procedure for solving the problem. In [9] Chotova investigated the effect of a uniformly distributed inplane compressive force system - exerted on the outer boundary of the plate - on the natural frequencies of circular plates assuming axisymmetric behavior. Numerical solutions were found by determining the zeros of the corresponding frequency determinant, which is regarded as a function of the load. It is also important to cite papers [10, 11] by Chen and his co-authors who dealt with the large amplitude vibrations of an initially stressed thick circular plate. They applied energetic methods in order to clarify how the load influences the vibrations. The results achieved are presented graphically. Paper [12] is devoted to the stability of parametric vibrations of circular plates subjected to in-plane forces by using the Liapunov method. Younesian [13] studied the forced vibrations of annular plates under the action of a transverse load rotating on the outer boundary.

The main objective of the present paper is to clarify mathematically - by setting up polynomial relationships - how the constant in plane load influences the vibrations of clamped, simply supported and spring supported circular plates. We attack the problem by determining the Green functions of the prestressed plates, which might also be useful for solving various boundary value problems in a closed form if the plates are subjected to transverse loads. With the knowledge of the Green functions the two parameter eigenvalue problems established for the natural frequencies are reduced to eigenvalue problems governed by homogenous Fredholm integral equations with symmetric kernels. These formulations are basically the same as those of the boundary element methods; however the kernels are not singular. After having set up the eigenvalue problems this way we solve them by the boundary element method.

The paper is organized into seven sections. Section 2 is a collection of the most important notations and notational conventions. Section 3 presents the governing 
equations of the problem raised. Section 4 outlines the determination of the Green functions. It is also shown that the third Green function contains the other two at the limit, i.e., if the spring constant tends to zero or infinity. The algorithm of the numerical solutions is considered in Section 5. Computational and analytical (closed form approximate) solutions are presented in Section 6. The last section is a conclusion. We remark that some preliminary results were published earlier in Hungarian [14, 2007].

\section{Notations And notational CONVEntions}

The table below presents the most important notations and notational conventions.

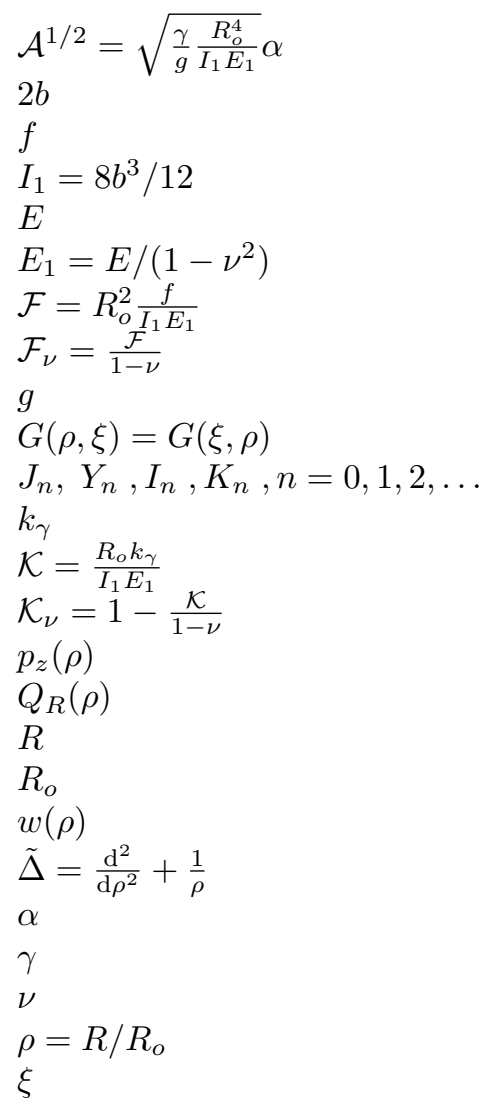

dimensionless natural frequency

thickness of the plate

radial load in the mid-plane

moment of inertia

Young's modulus

modified Young's modulus

dimensionless in-plane load

modified dimensionless in-plane load

gravitational acceleration

Green function

Bessel functions

spring constant

dimensionless spring constant

modified dimensionless spring constant

load in the $z$ direction

shear force

radius

outer radius of the plate

deflection

differential operator

natural frequency

density

Poisson number

dimensionless independent variable

dimensionless independent variable

The text contains further notations defined at the place of their first appearance. 


\section{Governing EQUATions}

If a circular plate is subjected to a constant radial load $f$ in its plane then the deflection $w$ due to the load $p_{z}(\rho)$ acting perpendicularly to the middle plane of the plate should meet the differential equation

$$
\tilde{\Delta} \tilde{\Delta} w \pm \mathcal{F} \tilde{\Delta} w=\frac{R_{o}^{4}}{I_{1} E_{1}} p_{z}, \quad \tilde{\Delta}=\frac{\mathrm{d}^{2}}{\mathrm{~d} \rho^{2}}+\frac{1}{\rho} \frac{\mathrm{d}}{\mathrm{d} \rho}
$$

if axisymmetric deformations are assumed - the sign preceding $\mathcal{F}$ is positive for compression and negative for tension - Figure 1 shows a compressive load [15, 2003]. Depending on what the supports are equation (3.1) should be associated with appropriate boundary conditions. As regards the outer boundary it is clear from Figure 1 that for a clamped plate

$$
\left.w\right|_{\rho=1}=0,\left.\quad \frac{\mathrm{d} w}{\mathrm{~d} \rho}\right|_{\rho=1}=0,
$$

are the boundary conditions. If the plate is simply supported then the boundary conditions are of the form

$$
\left.w\right|_{\rho=1}=0,\left.\quad\left(\frac{\mathrm{d}^{2} w}{\mathrm{~d} \rho^{2}}+\frac{\nu}{\rho} \frac{\mathrm{d} w}{\mathrm{~d} \rho}\right)\right|_{\rho=1}=0 .
$$

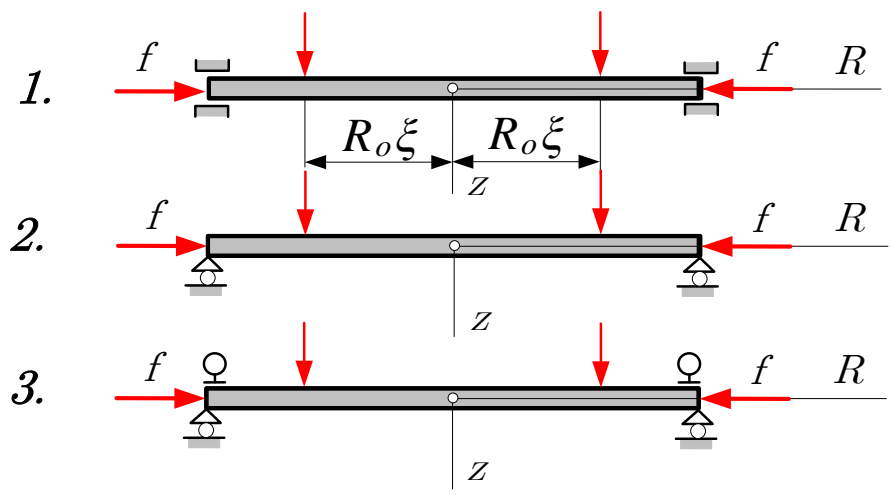

Figure 1.

Finally if the plate is supported by a torsional spring which exerts a bending moment on the boundary of the plate then

$$
w=0,\left.\quad\left(\frac{\mathrm{d}^{2} w}{\mathrm{~d} \rho^{2}}+\frac{\nu}{\rho} \frac{\mathrm{d} w}{\mathrm{~d} \rho}\right)\right|_{\rho=1}=-\left.\frac{R_{o}}{I_{1} E_{1}} k_{\gamma} \frac{\mathrm{d} w}{\mathrm{~d} \rho}\right|_{\rho=1}
$$

are the boundary conditions. It is also clear that the deflection at the center of the plate should meet the conditions

$$
\left.w\right|_{\rho=0}=\text { finite },\left.\quad \frac{\mathrm{d} w}{\mathrm{~d} \rho}\right|_{\rho=0}=0
$$


as well. For our later considerations we remark that the shear force $Q_{R}$ is related to the deflection via equation

$$
Q_{R} \frac{R_{o}^{3}}{I_{1} E_{1}}=\frac{\mathrm{d}}{\mathrm{d} \rho}\left[\frac{\mathrm{d}^{2}}{\mathrm{~d} \rho^{2}}+\frac{1}{\rho} \frac{\mathrm{d}}{\mathrm{d} \rho} \pm \mathcal{F}\right] w(\rho) .
$$

We remark that the boundary conditions and the formula for the shear force are all taken from Kozák [16].

\section{Green Functions}

4.1. Definition of the Green function. Assume that the plate is subjected to a uniform load distributed on the circle with radius $R_{o} \xi$ ( $\xi$ is also a dimensionless coordinate) - see Figure 1. The resultant of the total load is assumed to be 1 . The deflection due to the load at $\rho$ is denoted by $G(\rho, \xi)$ and is referred to as the Green function. Observe that the Green function should satisfy the homogenous equation in 3.1 if $0 \leq \rho<\xi$ and $\xi<\rho \leq 1$.

\subsection{Green functions for a compressive $f$.}

4.2.1. General solution. As is well known the general solution of the homogenous equation in (3.1) assumes the form

$$
w(\rho)=c_{1}+c_{2} \ln \rho+c_{3} J_{o}(\sqrt{\mathcal{F}} \rho)+c_{4} Y_{o}(\sqrt{\mathcal{F}} \rho),
$$

where $c_{1}, c_{2}, c_{3}$ and $c_{4}$ are undetermined constants of integration.

Since the Green function should meet conditions 3.5 it assumes the form

$$
\begin{aligned}
& G(\rho, \xi)=A_{1}+A_{3} J_{0}(\sqrt{\mathcal{F}} \rho), \quad 0 \leq \rho \leq \xi, \\
& G(\rho, \xi)=B_{1}+B_{2} \ln \rho+B_{3} J_{0}(\sqrt{\mathcal{F}} \rho)+B_{4} Y_{0}(\sqrt{\mathcal{F}} \rho), \quad \xi \leq \rho \leq 1,
\end{aligned}
$$

where the constants of integration $A_{1}, A_{3}$ and $B_{1}, B_{2}, B_{3}, B_{4}$ are to be determined from the continuity and discontinuity conditions prescribed at $\rho=\xi$ and form the boundary conditions which are imposed on the boundary $\rho=1$.

Observe that the continuity conditions

$$
\begin{aligned}
G(\xi-0, \xi) & =G(\xi+0, \xi), \\
G^{\prime}(\xi-0, \xi) & =G^{\prime}(\xi+0, \xi), \\
G^{\prime \prime}(\xi-0, \xi) & =G^{\prime \prime}(\xi+0, \xi)
\end{aligned}
$$

and the discontinuity condition

$$
2 \pi R_{o} \xi\left[Q_{R}(\xi+0)-Q_{R}(\xi-0)\right]=2 \pi R_{o} \xi Q_{R}(\xi+0)=1,
$$

in which $Q_{R}(\xi-0)=0$ from the vertical equilibrium, are all independent of the supports. Here (a) derivatives with respect to $\rho$ are denoted by primes and (b) it follows from $(3.6)$ that

$$
Q_{R} \frac{R_{o}^{3}}{I_{1} E_{1}}=\frac{\mathrm{d}}{\mathrm{d} \rho}\left[\frac{\mathrm{d}^{2}}{\mathrm{~d} \rho^{2}}+\frac{1}{\rho} \frac{\mathrm{d}}{\mathrm{d} \rho}+\mathcal{F}\right] G(\xi, \rho) .
$$


4.2.2. Green function for the clamped plate. If the plate is clamped the continuity and discontinuity conditions are associated with the boundary conditions

$$
G(1, \xi)=0, \quad G^{\prime}(1, \xi)=0 .
$$

In what follows we need the derivatives

$$
\begin{aligned}
& G^{\prime}(\rho, \xi)=-A_{3} \sqrt{\mathcal{F}} J_{1}(\sqrt{\mathcal{F}} \rho) \quad 0 \leq \rho \leq \xi \\
& G^{\prime}(\rho, \xi)=B_{2} \frac{1}{\rho}-B_{3} \sqrt{\mathcal{F}} J_{1}(\sqrt{\mathcal{F}} \rho)-B_{4} \sqrt{\mathcal{F}} Y_{1}(\sqrt{\mathcal{F}} \rho) \quad \xi \leq \rho \leq 1, \\
& G^{\prime \prime}(\rho, \xi)=A_{3} \mathcal{F}\left[\frac{J_{1}(\sqrt{\mathcal{F}} \rho)}{\sqrt{\mathcal{F}} \rho}-J_{0}(\sqrt{\mathcal{F}} \rho)\right] \quad 0 \leq \rho \leq \xi \\
& G^{\prime \prime}(\rho, \xi)=-B_{2} \frac{1}{\rho^{2}}+B_{3} \mathcal{F}\left[\frac{J_{1}(\sqrt{\mathcal{F}} \rho)}{\sqrt{\mathcal{F}} \rho}-J_{0}(\sqrt{\mathcal{F}} \rho)\right]+ \\
& \quad+B_{4} \mathcal{F}\left(\frac{Y_{1}(\sqrt{\mathcal{F}} \rho)}{\sqrt{\mathcal{F}} \rho}-Y_{0}(\sqrt{\mathcal{F}} \rho)\right) \quad \xi \leq \rho \leq 1, \\
& G^{\prime \prime \prime}(\rho, \xi)=-A_{3} \frac{\mathcal{F}^{3 / 2}}{4}\left[J_{3}(\sqrt{\mathcal{F}} \rho)-3 J_{1}(\sqrt{\mathcal{F}} \rho)\right]= \\
& =A_{2} \frac{\mathcal{F}^{3 / 2}}{4}\left[\frac{1}{\sqrt{\mathcal{F}} \rho} J_{2}(\sqrt{\mathcal{F}} \rho)-J_{1}(\sqrt{\mathcal{F}} \rho)\right] \quad 0 \leq \rho \leq \xi
\end{aligned}
$$

and

$$
\begin{aligned}
G^{\prime \prime \prime}(\rho, \xi)= & \frac{2}{\rho^{3}} B_{2}-B_{3} \frac{\mathcal{F}^{3 / 2}}{4}\left[J_{3}(\sqrt{\mathcal{F}} \rho)-3 J_{1}(\sqrt{\mathcal{F}} \rho)\right]- \\
& -B_{4} \frac{\mathcal{F}^{3 / 2}}{4}\left[Y_{3}(\sqrt{\mathcal{F}} \rho)-3 Y_{1}(\sqrt{\mathcal{F}} \rho)\right]= \\
= & \frac{2}{\rho^{3}} B_{2}+B_{3} \frac{\mathcal{F}^{3 / 2}}{4}\left[\frac{1}{\sqrt{\mathcal{F}} \rho} J_{2}(\sqrt{\mathcal{F}} \rho)-J_{1}(\sqrt{\mathcal{F}} \rho)\right]+ \\
& +B_{4} \frac{\mathcal{F}^{3 / 2}}{4}\left[\frac{1}{\sqrt{\mathcal{F}} \rho} Y_{2}(\sqrt{\mathcal{F}} \rho)-Y_{1}(\sqrt{\mathcal{F}} \rho)\right] \quad \xi \leq \rho \leq 1
\end{aligned}
$$

obtained by using relations A.1.

Substituting the Green function and its derivatives into the continuity conditions (4.3a) and then combining the continuity conditions $4.3 \mathrm{~b}$ and $4.3 \mathrm{c}$ we have

$$
\begin{aligned}
A_{1}+A_{3} J_{0}(\sqrt{\mathcal{F}} \xi) & =B_{1}+B_{2} \ln \xi+B_{3} J_{0}(\sqrt{\mathcal{F}} \xi)+B_{4} Y_{0}(\sqrt{\mathcal{F}} \xi) \\
-A_{3} \sqrt{\mathcal{F}} J_{1}(\sqrt{\mathcal{F}} \xi) & =B_{2} \frac{1}{\xi}-B_{3} \sqrt{\mathcal{F}} J_{1}(\sqrt{\mathcal{F}} \xi)-B_{4} \sqrt{\mathcal{F}} Y_{1}(\sqrt{\mathcal{F}} \xi) \\
A_{3} J_{0}(\sqrt{\mathcal{F}} \xi) & =B_{3} J_{0}(\sqrt{\mathcal{F}} \xi)+B_{4} Y_{0}(\sqrt{\mathcal{F}} \xi) .
\end{aligned}
$$


It follows from 4.5 that

$$
\begin{aligned}
\frac{R_{K}^{3}}{I_{1} E_{1}} Q_{R}(\xi+0) & =\left.B_{2} \frac{d}{d \rho}\left\{-\frac{1}{\rho^{2}}+\frac{1}{\rho^{2}}+\mathcal{F} \ln \rho\right\}\right|_{\rho=\xi}+ \\
& +\left.B_{3} \frac{d}{d \rho}\left\{\left[\frac{\mathcal{F}}{2}\left[J_{2}(\sqrt{\mathcal{F}} \rho)-J_{0}(\sqrt{\mathcal{F}} \rho)\right]-\mathcal{F} \frac{J_{1}(\sqrt{\mathcal{F}} \rho)}{\sqrt{\mathcal{F}} \rho}\right]+\mathcal{F} J_{0}(\sqrt{\mathcal{F}} \rho)\right\}\right|_{\rho=\xi} \\
& +\left.B_{4} \frac{d}{d \rho}\left\{\left[\frac{\mathcal{F}}{2}\left[Y_{2}(\sqrt{\mathcal{F}} \rho)-Y_{0}(\sqrt{\mathcal{F}} \rho)\right]-\mathcal{F} \frac{Y_{1}(\sqrt{\mathcal{F}} \rho)}{\sqrt{\mathcal{F}} \rho}\right]+\mathcal{F} Y_{0}(\sqrt{\mathcal{F}} \rho)\right\}\right|_{\rho=\xi}= \\
& =\left.B_{2} \frac{d}{d \rho}\left\{-\frac{1}{\rho^{2}}+\frac{1}{\rho^{2}}+\mathcal{F} \ln \rho\right\}\right|_{\rho=\xi}+ \\
& +\left.B_{3} \frac{d}{d \rho} \underbrace{\left\{\left[\frac{\mathcal{F}}{2}\left[J_{2}(\sqrt{\mathcal{F}} \rho)+J_{0}(\sqrt{\mathcal{F}} \rho)\right]-\mathcal{F} \frac{J_{1}(\sqrt{\mathcal{F}} \rho)}{\sqrt{\mathcal{F}} \rho}\right]\right\}}_{=\text {zero }}\right|_{\rho=\xi} \\
& +\left.B_{4} \frac{d}{d \rho} \underbrace{\left\{\left[\frac{\mathcal{F}}{2}\left[Y_{2}(\sqrt{\mathcal{F}} \rho)+Y_{0}(\sqrt{\mathcal{F}} \rho)\right]-\mathcal{F} \frac{Y_{1}(\sqrt{\mathcal{F}} \rho)}{\sqrt{\mathcal{F}} \rho}\right]\right\}}_{=\text {zero }}\right|_{\rho=\xi}=B_{2} \mathcal{F} \frac{1}{\xi} .
\end{aligned}
$$

Consequently discontinuity condition (4.4) leads to the equation

$$
B_{2}=\frac{R_{o}^{3}}{I_{1} E_{1}} \frac{1}{2 \pi \xi R_{o}} \frac{1}{\mathcal{F}} \xi=\frac{R_{o}^{2}}{I_{1} E_{1} \frac{f R_{o}^{2}}{I_{1} E_{1}}} \frac{1}{2 \pi \xi} \xi=\frac{1}{2 \pi f} .
$$

The last two equations for the integration constants are obtained from the boundary conditions 4.6)

$$
\begin{aligned}
B_{1}+B_{3} J_{0}(\sqrt{\mathcal{F}})+B_{4} Y_{0}(\sqrt{\mathcal{F}}) & =0, \\
B_{2}-B_{3} \sqrt{\mathcal{F}} J_{1}(\sqrt{\mathcal{F}})-B_{4} \sqrt{\mathcal{F}} Y_{1}(\sqrt{\mathcal{F}}) & =0 .
\end{aligned}
$$

Introducing the notations $J_{n \rho}=J_{n}(\sqrt{\mathcal{F}} \rho), J_{n \xi}=J_{n}(\sqrt{\mathcal{F}} \xi), J_{n 1}=J_{n}(\sqrt{\mathcal{F}})$ and $Y_{n \rho}=Y_{n}(\sqrt{\mathcal{F}} \rho), Y_{n \xi}=Y_{n}(\sqrt{\mathcal{F}} \xi), Y_{n 1}=Y_{n}(\sqrt{\mathcal{F}})$ we can rewrite equations 4.10 in the following form:

$$
\left[\begin{array}{cccccc}
1 & J_{0 \xi} & -1 & -\ln \xi & -J_{0 \xi} & -Y_{0 \xi} \\
0 & -\sqrt{\mathcal{F}} J_{1 \xi} & 0 & -\frac{1}{\xi} & \sqrt{\mathcal{F}} J_{1 \xi} & \sqrt{\mathcal{F}} Y_{1 \xi} \\
0 & J_{0 \xi} & 0 & 0 & -J_{0 \xi} & -Y_{0 \xi} \\
0 & 0 & 0 & 1 & 0 & 0 \\
0 & 0 & 1 & 0 & J_{01} & Y_{01} \\
0 & 0 & 0 & 1 & -\sqrt{\mathcal{F}} J_{11} & -\sqrt{\mathcal{F}} Y_{11}
\end{array}\right]\left[\begin{array}{c}
A_{1} \\
A_{3} \\
B_{1} \\
B_{2} \\
B_{3} \\
B_{4}
\end{array}\right]=\left[\begin{array}{c}
0 \\
0 \\
0 \\
\frac{1}{2 \pi f} \\
0 \\
0
\end{array}\right]
$$

After solving equation system 4.11) and substituting solutions (B.1) given in the Appendix into (4.2) we have 


$$
\begin{array}{r}
G(\rho, \xi)=\frac{J_{11} \ln \xi+\frac{1}{\sqrt{\mathcal{F}}}\left(J_{0 \rho}+J_{0 \xi}-J_{01}\right)+\frac{\pi}{2} J_{0 \rho}\left(J_{0 \xi} Y_{11}-Y_{0 \xi} J_{11}\right)}{2 \pi f J_{11}} \quad 0 \leq \rho \leq \xi, \\
G(\rho, \xi)=\frac{J_{11} \ln \rho+\frac{1}{\sqrt{\mathcal{F}}}\left(J_{0 \xi}+J_{0 \rho}-J_{01}\right)+\frac{\pi}{2} J_{0 \xi}\left(J_{0 \rho} Y_{11}-Y_{0 \rho} J_{11}\right)}{2 \pi f J_{11}} \quad \xi \leq \rho \leq 1,
\end{array}
$$

which is the Green function for the clamped plate. It can be checked with ease that the Green function is symmetric, i.e., $G(\rho, \xi)=G(\xi, \rho)$.

4.2.3. Green function for the simply supported plate. If the plate is simply supported the continuity and discontinuity conditions are associated with the following boundary conditions:

$$
G(1, \xi)=0, \quad G^{\prime \prime}(1, \xi)+\frac{\nu}{\rho} G^{\prime}(1, \xi)=0
$$

Observe that only one equation differs from those we used earlier - compare $4.13{ }_{2}$ and $(4.6)_{2}$. For the sake of brevity we omit the paper and pencil calculations and present the final result only:

$$
\begin{gathered}
G(\rho, \xi)=\frac{J_{01}-J_{0 \xi}-J_{0 \rho}+\frac{\pi}{2} J_{0 \xi} J_{0 \rho}\left(\mathcal{F}_{\nu} Y_{01}-\sqrt{\mathcal{F}} Y_{11}\right)}{2 \pi f\left(\mathcal{F}_{\nu} J_{01}-\sqrt{\mathcal{F}} J_{11}\right)}+ \\
+\frac{\ln \xi-\frac{\pi}{2} J_{0 \rho} Y_{0 \xi}}{2 \pi f} \quad 0 \leq \rho \leq \xi, \\
G(\rho, \xi)=\frac{J_{01}-J_{0 \rho}-J_{0 \xi}+\frac{\pi}{2} J_{0 \rho} J_{0 \xi}\left(\mathcal{F}_{\nu} Y_{01}-\sqrt{\mathcal{F}} Y_{11}\right)}{4 \pi f\left(\mathcal{F}_{\nu} J_{01}-\sqrt{\mathcal{F}} J_{11}\right)}+ \\
+\frac{\ln \rho-\frac{\pi}{2} J_{0 \xi} Y_{0 \rho}}{2 \pi f} \quad \xi \leq \rho \leq 1 .
\end{gathered}
$$

This Green function is also symmetric, i.e., $G(\rho, \xi)=G(\xi, \rho)$.

4.2.4. Green function for the spring-supported plate. For the spring-supported plate the continuity and discontinuity conditions are associated with the following boundary conditions:

$$
G(1, \xi)=0, \quad G(1, \xi)^{\prime \prime}+\frac{\nu}{\rho} G(1, \xi)^{\prime}+\frac{R_{o}}{I_{1} E_{1}} k_{\gamma} G(1, \xi)^{\prime}=0 .
$$

Without entering into details we have arrived at the following Green function

$$
\begin{gathered}
G(\rho, \xi)=\frac{\mathcal{K}_{\nu}\left(J_{01}-J_{0 \xi}-J_{0 \rho}\right)+\frac{\pi}{2} J_{0 \rho} J_{0 \xi}\left(\mathcal{F}_{\nu} Y_{01}-\sqrt{\mathcal{F}} \mathcal{K}_{\nu} Y_{11}\right)}{2 \pi f\left(\mathcal{F}_{\nu} J_{01}-\sqrt{\mathcal{F}} \mathcal{K}_{\nu} J_{11}\right)}+ \\
+\frac{\ln \xi-\frac{\pi}{2} J_{0 \rho} Y_{0 \xi}}{2 \pi f} \quad 0 \leq \rho \leq \xi
\end{gathered}
$$




$$
\begin{gathered}
G(\rho, \xi)=\frac{\mathcal{K}_{\nu}\left(J_{01}-J_{0 \rho}-J_{0 \xi}\right)+\frac{\pi}{2} J_{0 \xi} J_{0 \rho}\left(\mathcal{F}_{\nu} Y_{01}-\sqrt{\mathcal{F}} \mathcal{K}_{\nu} Y_{11}\right)}{2 \pi f\left(\mathcal{F}_{\nu} J_{01}-\sqrt{\mathcal{F}} \mathcal{K}_{\nu} J_{11}\right)}+ \\
+\frac{\ln \rho-\frac{\pi}{2} J_{0 \xi} Y_{0 \rho}}{2 \pi f} \quad \xi \leq \rho \leq 1
\end{gathered}
$$

In the formulae providing the last two Green functions

$$
\mathcal{K}=\frac{R_{o} k_{\gamma}}{I_{1} E_{1}}, \quad \mathcal{F}_{\nu}=\frac{\mathcal{F}}{1-\nu}, \quad \mathcal{K}_{\nu}=1-\frac{\mathcal{K}}{1-\nu} .
$$

It can be shown that (a) for $\mathcal{K} \rightarrow 0$ the Green function 4.16 coincides with the Green function (4.14) valid for the simply supported plate and (b) for $\mathcal{K} \rightarrow \infty$ the Green function (4.16) coincides with the Green function 4.12 valid for the clamped plate.

\subsection{Green functions for a tensile $f$.}

4.3.1. General solution. If the in-plane load is a tensile load

$$
w(\rho)=c_{1}+c_{2} \ln \rho+c_{3} I_{0}(\sqrt{\mathcal{F}} \rho)+c_{4} K_{0}(\sqrt{\mathcal{F}} \rho)
$$

is the general solution we need when determining the Green function $-c_{1}, c_{2}, c_{3}$ and $c_{4}$ are again undetermined constants of integration. With the knowledge of the general solution we assume that the Green function, which satisfies conditions (3.5), can be given in the following form

$$
\begin{array}{ll}
G(\rho, \xi) & =A_{1}+A_{3} I_{0}(\sqrt{\mathcal{F}} \rho), \quad \rho<\xi, \\
G(\rho, \xi) & =B_{1}+B_{2} \ln \rho+B_{3} I_{0}(\sqrt{\mathcal{F}} \rho)+B_{4} K_{0}(\sqrt{\mathcal{F}} \rho), \quad \rho>\xi
\end{array}
$$

in which the integration constants are denoted in the same way as for the case of compressive $f$ - however this may not cause any misunderstanding.

4.3.2. Green function for the clamped plate. When determining the Green function we need the following derivatives

$$
\begin{array}{rlr}
G^{\prime}(\rho, \xi) & =A_{3} \sqrt{\mathcal{F}} I_{1}(\sqrt{\mathcal{F}} \rho) \quad \rho<\xi \\
G^{\prime}(\rho, \xi) & =B_{2} \frac{1}{\rho}+B_{3} \sqrt{\mathcal{F}} I_{1}(\sqrt{\mathcal{F}} \rho)-B_{4} \sqrt{\mathcal{F}} K_{1}(\sqrt{\mathcal{F}} \rho) & \rho>\xi \\
G^{\prime \prime}(\rho, \xi) & =A_{3} \mathcal{F}\left(I_{0}(\sqrt{\mathcal{F}} \rho)-\frac{1}{\sqrt{\mathcal{F}} \rho} I_{1}(\sqrt{\mathcal{F}} \rho)\right) & \rho<\xi \\
G^{\prime \prime}(\rho, \xi) & =-B_{2} \frac{1}{\rho^{2}}+B_{3} \mathcal{F}\left(I_{0}(\sqrt{\mathcal{F}} \rho)-\frac{1}{\sqrt{\mathcal{F}} \rho} I_{1}(\sqrt{\mathcal{F}} \rho)\right)+ \\
& +B_{4} \mathcal{F}\left(K_{0}(\sqrt{\mathcal{F}} \rho)+\frac{1}{\sqrt{\mathcal{F}} \rho} K_{1}(\sqrt{\mathcal{F}} \rho)\right) & \rho>\xi \\
G^{\prime \prime \prime}(\rho, \xi) & =A_{3} \frac{\mathcal{F}^{3 / 2}}{4}\left[3 I_{1}(\sqrt{\mathcal{F}} \rho)+I_{3}(\sqrt{\mathcal{F}} \rho)\right]= &
\end{array}
$$




$$
\begin{aligned}
G^{\prime \prime \prime}(\rho, \xi) & =-2 B_{2} \frac{1}{\rho^{3}}+B_{3} \frac{\mathcal{F}^{3 / 2}}{4}\left[3 I_{1}(\sqrt{\mathcal{F}} \rho)+I_{3}(\sqrt{\mathcal{F}} \rho)\right]- \\
& -B_{4} \frac{\mathcal{F}^{3 / 2}}{4}\left[3 K_{1}(\sqrt{\mathcal{F}} \rho)+K_{3}(\sqrt{\mathcal{F}} \rho)\right]= \\
& =-2 B_{2} \frac{1}{\rho^{3}}+B_{3} \mathcal{F}^{3 / 2}\left(\frac{1}{\sqrt{\mathcal{F}} x} I_{2}(\sqrt{\mathcal{F}} x)-I_{1}(\sqrt{\mathcal{F}} x)\right) \\
& -B_{4} \mathcal{F}^{3 / 2}\left[\frac{1}{\sqrt{\mathcal{F}} x} K_{2}(\sqrt{\mathcal{F}} x)+K_{1}(\sqrt{\mathcal{F}} x)\right] \quad \rho>\xi
\end{aligned}
$$

obtained by utilizing A.2 . Substituting the Green function and its derivatives into the continuity conditions (4.3a) and combining 4.3b and 4.3c we have

$$
\begin{aligned}
A_{1}+A_{3} I_{0}(\sqrt{\mathcal{F}} \xi) & =B_{1}+B_{2} \ln \xi+B_{3} I_{0}(\sqrt{\mathcal{F}} \xi)+B_{4} K_{0}(\sqrt{\mathcal{F}} \xi), \\
A_{3} \sqrt{\mathcal{F}} I_{1}(\sqrt{\mathcal{F}} \xi) & =B_{2} \frac{1}{\xi}+B_{3} \sqrt{\mathcal{F}} I_{1}(\sqrt{\mathcal{F}} \xi)-B_{4} \sqrt{\mathcal{F}} K_{1}(\sqrt{\mathcal{F}} \xi), \\
A_{3} I_{0}(\sqrt{\mathcal{F}} \xi) & =B_{3} I_{0}(\sqrt{\mathcal{F}} \xi)+B_{4} K_{0}(\sqrt{\mathcal{F}} \xi) .
\end{aligned}
$$

By repeating the line of thought resulting in equation (4.10d discontinuity condition (4.4) leads to the equation

$$
B_{2}=-1 / 2 \pi f \text {. }
$$

The last two equations for the integration constants are provided again by the boundary conditions 4.6

$$
\begin{aligned}
B_{1}+B_{3} I_{0}(\sqrt{\mathcal{F}})+B_{4} K_{0}(\sqrt{\mathcal{F}}) & =0, \\
B_{2}+B_{3} \sqrt{\mathcal{F}} I_{1}(\sqrt{\mathcal{F}})-B_{4} \sqrt{\mathcal{F}} K_{1}(\sqrt{\mathcal{F}}) & =0 .
\end{aligned}
$$

Let $I_{n \rho}=I_{n}(\sqrt{\mathcal{F}} \rho), I_{n \xi}=I_{n}(\sqrt{\mathcal{F}} \xi), I_{n 1}=I_{n}(\sqrt{\mathcal{F}}), K_{n \rho}=K_{n}(\sqrt{\mathcal{F}} \rho), K_{n \xi}=$ $K_{n}(\sqrt{\mathcal{F}} \xi)$ and $K_{n 1}=K_{n}(\sqrt{\mathcal{F}})$. Making use of the notations introduced equation system 4.23 can be cast into the following form:

$$
\left[\begin{array}{cccccc}
1 & I_{0 \xi} & -1 & -\ln \xi & -I_{0 \xi} & -K_{0 \xi} \\
0 & \sqrt{\mathcal{F}} J_{1 \xi} & 0 & -\frac{1}{\xi} & -\sqrt{\mathcal{F}} I_{1 \xi} & \sqrt{\mathcal{F}} K_{1 \xi} \\
0 & I_{0 \xi} & 0 & 0 & -I_{0 \xi} & -K_{0 \xi} \\
0 & 0 & 0 & 1 & 0 & 0 \\
0 & 0 & 1 & 0 & I_{01} & K_{01} \\
0 & 0 & 0 & 1 & \sqrt{\mathcal{F}} I_{11} & -\sqrt{\mathcal{F}} K_{11}
\end{array}\right]\left[\begin{array}{c}
A_{1} \\
A_{3} \\
B_{1} \\
B_{2} \\
B_{3} \\
B_{4}
\end{array}\right]=\left[\begin{array}{c}
0 \\
0 \\
0 \\
-\frac{1}{2 \pi f} \\
0 \\
0
\end{array}\right]
$$

With the integration constants $A_{1}, A_{3}, B_{1}, \ldots, B_{4}$ equation 4.19 yields the Green function as

$$
G(\rho, \xi)=\frac{I_{11} \ln \xi+\frac{1}{\sqrt{F}}\left(I_{01}-I_{0 \xi}-I_{0 \rho}\right)+I_{0 \rho}\left(I_{0 \xi} K_{11}+K_{0 \xi} I_{11}\right)}{2 I_{11} \pi f} \quad 0<\rho \leq \xi,
$$




$$
G(\xi, \rho)=\frac{I_{11} \ln \rho+\frac{1}{\sqrt{F}}\left(I_{01}-I_{0 \rho}-I_{0 \xi}\right)+I_{0 \xi}\left(I_{0 \rho} K_{11}+K_{0 \rho} I_{11}\right)}{2 I_{11} \pi f} \quad \xi \leq \rho \leq 1 .
$$

4.3.3. Green function for the simply supported plate. We present the Green function without providing details concerning the paper and pencil calculations

$$
\begin{gathered}
G(\rho, \xi)=\frac{I_{0 \xi}+I_{0 \rho}-I_{01}-I_{0 \rho} I_{0 \xi}\left(\sqrt{\mathcal{F}} K_{11}+\mathcal{F}_{\nu} K_{01}\right)}{2 \pi f\left(\mathcal{F}_{\nu} I_{01}-\sqrt{\mathcal{F}} I_{11}\right)}+ \\
+\frac{\ln \xi+I_{0 \rho} K_{0 \xi}}{2 \pi f} \quad 0<\rho \leq \xi, \\
G(\rho, \xi)=\frac{I_{0 \rho}+I_{0 \xi}-I_{01}-I_{0 \xi} I_{0 \rho}\left(\sqrt{\mathcal{F}} K_{11}-\mathcal{F}_{\nu} K_{01}\right)}{2 \pi f\left(\mathcal{F}_{\nu} I_{01}-\sqrt{\mathcal{F}} I_{11}\right)}+ \\
+\frac{\ln \rho+I_{0 \xi} K_{0 \rho}}{2 \pi f} \quad \xi \leq \rho \leq 1 .
\end{gathered}
$$

4.3.4. Green function for the spring supported plate. The Green function is given below again without presenting the calculations

$$
\begin{gathered}
G(\rho, \xi)=\frac{\mathcal{K}_{\nu}\left(I_{0 \xi}+I_{0 \rho}-I_{01}\right)-I_{0 \rho} I_{0 \xi}\left(\mathcal{F}_{\nu} K_{01}+\sqrt{\mathcal{F}} \mathcal{K}_{\nu} K_{11}\right)}{2 \pi f\left(\mathcal{F}_{\nu} I_{01}-\sqrt{\mathcal{F}} \mathcal{K}_{\nu} I_{11}\right)}+ \\
+\frac{\ln \xi+I_{0 \rho} K_{0 \xi}}{2 \pi f} \quad 0<\rho \leq \xi, \\
G(\xi, \rho)=\frac{\mathcal{K}_{\nu}\left(I_{0 \rho}+I_{0 \xi}-I_{01}\right)-I_{0 \xi} I_{0 \rho}\left(\mathcal{F}_{\nu} K_{01}+\sqrt{\mathcal{F}} \mathcal{K}_{\nu} K_{11}\right)}{2 \pi f\left(\mathcal{F}_{\nu} I_{01}-\sqrt{\mathcal{F}} \mathcal{K}_{\nu} I_{11}\right)}+ \\
+\frac{\ln \rho+K_{0 \rho} I_{0 \xi}}{2 \pi f} \quad \xi \leq \rho \leq 1 .
\end{gathered}
$$

If $\mathcal{K} \rightarrow 0$ 4.27) coincides with 4.25. Similarly if $\mathcal{K} \rightarrow \infty$ 4.27) coincides with (4.26.

4.4. Solutions of statical boundary value problems. Given the Green functions, the deflection due to an axisymmetric load $p_{z}(\rho)$ can always be calculated as

$$
w(\rho)=2 \pi R_{o}^{2} \int_{0}^{1} G(\rho, \xi) p_{z}(\xi) \xi \mathrm{d} \xi .
$$




\section{INTEGRAL EQUATION FOR THE NATURAL FREQUENCIES}

5.1. Integral equation of the problem. Under the assumption of harmonic vibrations the amplitude $W(\rho)$ of the vibrations $w(\rho, t)$ should satisfy the differential equation

$$
\left(\frac{\mathrm{d}^{2}}{\mathrm{~d} \rho^{2}}+\frac{1}{\rho} \frac{\mathrm{d}}{\mathrm{d} \rho}\right)\left[\left(\frac{\mathrm{d}^{2}}{\mathrm{~d} \rho^{2}}+\frac{1}{\rho} \frac{\mathrm{d}}{\mathrm{d} \rho}\right) W+\mathcal{F} W\right]=\frac{R_{o}^{4}}{I_{1} E_{1}} \frac{\gamma}{g} \alpha^{2} W
$$

where $\gamma$ is the plate weight for the unit area of the middle surface and $g$ is the gravitational acceleration. The eigenvalue problems defined by equation (5.1) and boundary conditions (3.2), 3.3) and (3.4) are all self adjoint. Since $\alpha^{2} \gamma W / g$ corresponds to $p_{z}$ in equation (3.1) it follows from equation 4.28) that the amplitude $W(\rho)$ should satisfy the integral equation

$$
W(\rho)=\lambda \int_{0}^{1} \hat{G}(\rho, \xi) W(\xi) \xi \mathrm{d} \xi \quad \text { where } \quad \lambda=\frac{\gamma}{g} \alpha^{2}, \quad \hat{G}(\rho, \xi)=2 \pi R_{o}^{2} G(\rho, \xi)
$$

which can be manipulated into the following form:

$$
W(\rho)=\frac{\gamma}{g} \alpha^{2} \int_{0}^{1} \frac{R_{o}^{2}}{f} \tilde{G}(\rho, \xi) W(\xi) \xi \mathrm{d} \xi=\underbrace{\frac{\gamma}{g} \frac{\alpha^{2} R_{o}^{4}}{I_{1} E_{1}}}_{\mathcal{A}} \int_{0}^{1} \frac{1}{\underbrace{\frac{f R_{o}^{2}}{I_{1} E_{1}}}_{\mathcal{F}}} \tilde{G}(\rho, \xi) W(\xi) \xi \mathrm{d} \xi .
$$

Here $\mathcal{A}$ and $\mathcal{F}$ are dimensionless quantities: $\mathcal{A}$ is proportional to the square of a natural frequency, $\mathcal{F}$ is proportional to the load. If we introduce a new unknown function

$$
y(\rho)=\sqrt{\rho} W(\rho)
$$

then we have

$$
\underbrace{\sqrt{\rho} W(\rho)}_{y(\rho)}=\mathcal{A} \int_{0}^{1} \underbrace{\sqrt{\rho} \frac{\tilde{G}(\rho, \xi)}{\mathcal{F}} \sqrt{(\xi)}}_{\mathcal{G}(\rho, \xi)} \underbrace{\sqrt{\xi} W(\xi)}_{y(\xi)} \mathrm{d} \xi, \text { labelVibr } 50
$$

that is

$$
y(\rho)=\mathcal{A} \int_{0}^{1} \mathcal{G}(\rho, \xi) y(\xi) \mathrm{d} \xi .
$$

The above equation is a homogenous Fredholm integral equation with a symmetric kernel. At the same time this equation is an eigenvalue problem with $\mathcal{A}$ as an eigenvalue, which is a function of the dimensionless in-plane load $\mathcal{F}$.

5.2. Computational algorithm. A numerical solution to the eigenvalue problem (5.6) can be sought by quadrature methods [17. Consider the integral formula

$$
J(\phi)=\int_{0}^{1} \phi(\xi) \mathrm{d} \xi \equiv \sum_{j=0}^{n} w_{j} \phi\left(\xi_{j}\right), \quad \xi_{j} \in[0,1], \quad j=0,1, \ldots, n,
$$


where $\phi(\xi)$ is a scalar and the weights $w_{j}$ are known. Making use of the above equation we obtain from $(5.6)$ that

$$
\sum_{j=0}^{n} w_{j} \mathcal{G}\left(\rho, \xi_{j}\right) y\left(\xi_{j}\right)=\chi y(\rho), \quad \chi=1 / \mathcal{A}, \quad x \in[0,1], \quad j=0,1, \ldots, n
$$

the solution of which yields an approximate eigenvalue $\mathcal{A}=1 / \chi$ and an approximate eigenfunction $y(\rho)$. After setting $\rho$ to $\rho_{i}(i=0,1,2, \ldots, n)$ we have

$$
\sum_{j=0}^{n} w_{j} \mathcal{G}\left(\rho_{i}, \xi_{j}\right) y\left(\xi_{j}\right)=\chi y\left(\rho_{i}\right) \quad \chi=1 / \mathcal{A} \quad x \in[0,1]
$$

or

$$
\mathcal{G} \mathcal{D} \tilde{y}=\chi \tilde{y}
$$

where $\mathcal{G}=\left[G\left(\rho_{i}, \xi_{j}\right)\right]$ is symmetric,

$$
\mathcal{D}=\operatorname{diag}\left(w_{0}|\ldots| w_{k}|\ldots| w_{n} \mid\right) \quad \text { and } \quad \tilde{y}^{T}=\left[y\left(x_{0}\right)\left|y\left(x_{1}\right)\right| \ldots \mid y\left(x_{n}\right)\right] .
$$

After solving the algebraic eigenvalue problem (5.10) we have the approximate eigenvalues $\chi_{r}$ and eigenvectors $\tilde{y}_{r}(r=0,1, \ldots, n)$. The corresponding eigenfunction is obtained by substituting back into equation (5.8):

$$
y(\rho)=\frac{1}{\chi_{r}} \sum_{j=0}^{n} w_{j} \mathcal{G}\left(\rho, \xi_{j}\right) y\left(\xi_{j}\right) .
$$

Divide the interval $[0,1]$ into equidistant subintervals of length $h$ and apply the integration formula to each subinterval. By repeating the line of thought leading to equation (5.10) one can show that the algebraic eigenvalue problem obtained is of the same structure as that of equation 5.10 .

It is also possible to attack the integral equation (5.1) as a boundary integral equation and to apply isoparametric approximation in the subintervals, i.e., on the elements. If this is the case one can approximate the eigenfunction on the $e$-th element (on the $e$-th subinterval which is mapped onto the interval $\eta \in[-1,1]$ and is denoted by $\left.L_{e}\right)$ as

$$
\stackrel{e}{y}(\eta)=\left[N_{1}(\eta)\left|N_{2}(\eta)\right| N_{3}(\eta)\right]\left[\begin{array}{c}
e \\
y_{1} \\
e \\
y_{2} \\
e \\
y_{3}
\end{array}\right]
$$

where quadratic local approximations are assumed,

$$
N_{1}=0.5 \eta(\eta-1), N_{2}=1-\eta^{2}, N_{3}=0.5 \eta(\eta+1),
$$

and $\stackrel{e}{y}_{i}$ is the value of the eigenfunction $y(\rho)$ at the left endpoint, the midpoint and the right endpoint of the element, respectively. Substituting equation 5.12 into equation (5.6) we have

$$
y(\rho)=\mathcal{A} \sum_{e=1}^{n_{b e}} \int_{\mathfrak{L}_{e}} \mathcal{G}[\rho, \xi(\eta)]\left[N_{1}(\eta)\left|N_{2}(\eta)\right| N_{3}(\eta)\right] \mathcal{J}(\eta) d \eta\left[\begin{array}{c}
e \\
y_{1} \\
y_{2} \\
e_{2} \\
y_{3}
\end{array}\right],
$$


where $n_{b e}$ is the number of elements (subintervals), $\mathcal{J}(\eta)$ is the Jacobian. Using equation (5.13) as our point of departure and repeating the line of thought leading to 5.10 we shall arrive again at an algebraic eigenvalue problem. A program has been developed in Fortran 90 which solves the algebraic eigenvalue problem formulated in this way.

\section{Computational Results}

6.1. Clamped plate. Let us introduce the following dimensionless quantities

$$
\mathcal{A}_{o i}=\frac{\gamma}{g} \frac{R_{o}^{2}}{I_{1} E_{1}} \alpha_{o i}^{2}, \quad \mathcal{A}_{i}=\frac{\gamma}{g} \frac{R_{o}^{2}}{I_{1} E_{1}} \alpha_{i}^{2}
$$

and

$$
\mathcal{F}_{o 1}=\frac{\gamma}{g} \frac{R_{o}^{2}}{I_{1} E_{1}} f_{1}
$$

where $\alpha_{o i}$ and $\alpha_{i}$ are the $i$-th $(i=1,2, \ldots)$ natural frequencies of the unloaded and loaded plates, while $f_{1}$ is the first critical load. The computational results for $i=1$ are presented in Table 1 .

TABLE 1

\begin{tabular}{|l|l|l|l|l|l|l|l|}
\hline $\mathcal{F} / \mathcal{F}_{o 1}$ & 0.068 & 0.136 & 0.204 & 0.272 & 0.341 & 0.409 & 0.477 \\
\hline $\mathcal{A} / \mathcal{A}_{o 1}-$ compression & 0.929 & 0.863 & 0.796 & 0.730 & 0.663 & 0.595 & 0.528 \\
$\mathcal{A} / \mathcal{A}_{o 1}-$ tension & 1.061 & 1.127 & 1.193 & 1.258 & 1.324 & 1.389 & 1.454 \\
\hline \hline $\mathcal{F} / \mathcal{F}_{o 1}$ & 0.545 & 0.613 & 0.681 & 0.749 & 0.817 & 0.886 & 0.954 \\
\hline $\mathcal{A} / \mathcal{A}_{o 1}-$ compression & 0.460 & 0.392 & 0.324 & 0.255 & 0.186 & 0.117 & 0.048 \\
$\mathcal{A} / \mathcal{A}_{o 1}-$ tension & 1.5189 & 1.584 & 1.648 & 1.713 & 1.777 & 1.841 & 1.906 \\
\hline
\end{tabular}

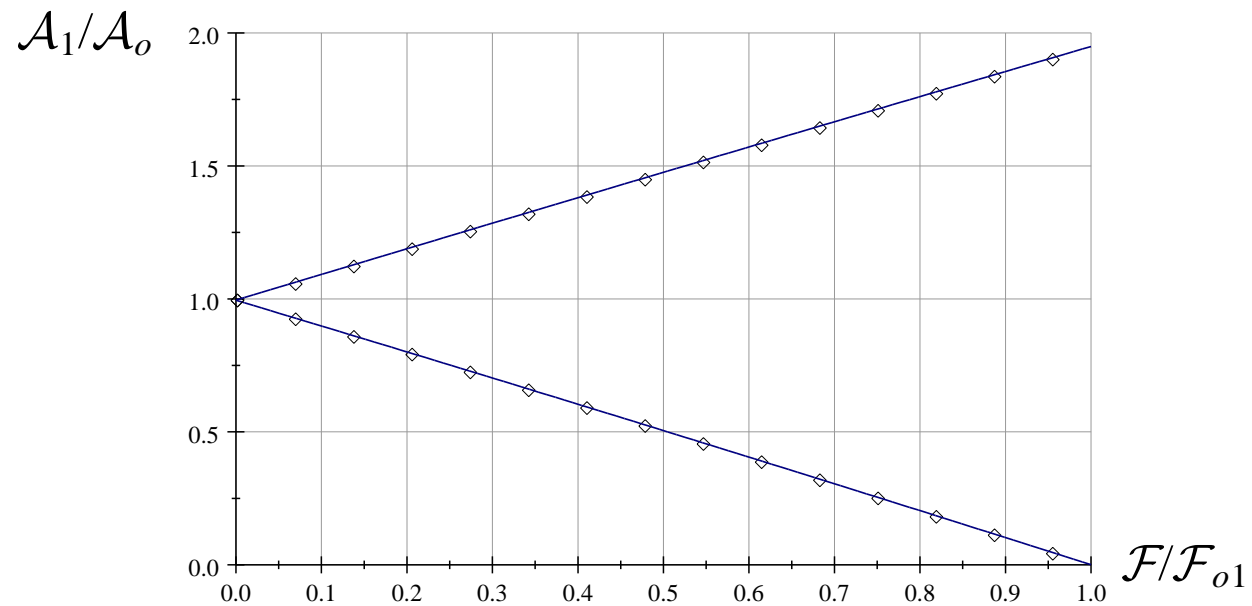

Figure 2. 
With the knowledge of computational results we have fitted the following curves onto the discrete points - these are denoted by diamonds in Figure $2\left(\mathcal{A}_{o 1}=104.85\right.$, $\left.\mathcal{F}_{o 1}=14.68\right)$

$$
\begin{aligned}
& \frac{\mathcal{A}_{1}}{\mathcal{A}_{o 1}}=0.99499-0.96594 \frac{\mathcal{F}}{\mathcal{F}_{o 1}}-2.8514 \times 10^{-2}\left(\frac{\mathcal{F}}{\mathcal{F}_{o 1}}\right)^{2} \\
& \frac{\mathcal{A}_{1}}{\mathcal{A}_{o 1}}=0.99561+0.96814 \frac{\mathcal{F}}{\mathcal{F}_{o 1}}-1.4742 \times 10^{-2}\left(\frac{\mathcal{F}}{\mathcal{F}_{o 1}}\right)^{2}
\end{aligned}
$$

Observe that the approximate solution 6.2 is practically linear in the interval $\mathcal{F} / \mathcal{F}_{o 1} \in$ $[0,1]$.

6.2. Simply supported plate. For a simply supported plate the computational results are presented in Table 2 under the assumption that $i=1$. These are denoted by diamonds in Figure 3 .

TABLE 2

\begin{tabular}{|l|l|l|l|l|l|l|l|}
\hline $\mathcal{F} / \mathcal{F}_{o 1}$ & 0.070 & 0.140 & 0.210 & 0.280 & 0.350 & 0.420 & 0.490 \\
\hline $\mathcal{A} / \mathcal{A}_{o 1}-$ compression & 0.930 & 0.860 & 0.790 & 0.720 & 0.650 & 0.580 & 0.510 \\
$\mathcal{A} / \mathcal{A}_{o 1}-$ tension & 1.071 & 1.141 & 1.211 & 1.281 & 1.351 & 1.421 & 1.491 \\
\hline \hline $\mathcal{F} / \mathcal{F}_{o 1}$ & 0.560 & 0.630 & 0.700 & 0.770 & 0.817 & 0.840 & 0.910 \\
\hline $\mathcal{A} / \mathcal{A}_{o 1}-$ compression & 0.440 & 0.370 & 0.300 & 0.230 & 0.159 & 0.089 & 0.019 \\
$\mathcal{A} / \mathcal{A}_{o 1}-$ tension & 1.561 & 1.631 & 1.701 & 1.771 & 1.841 & 1.911 & 1.981 \\
\hline
\end{tabular}

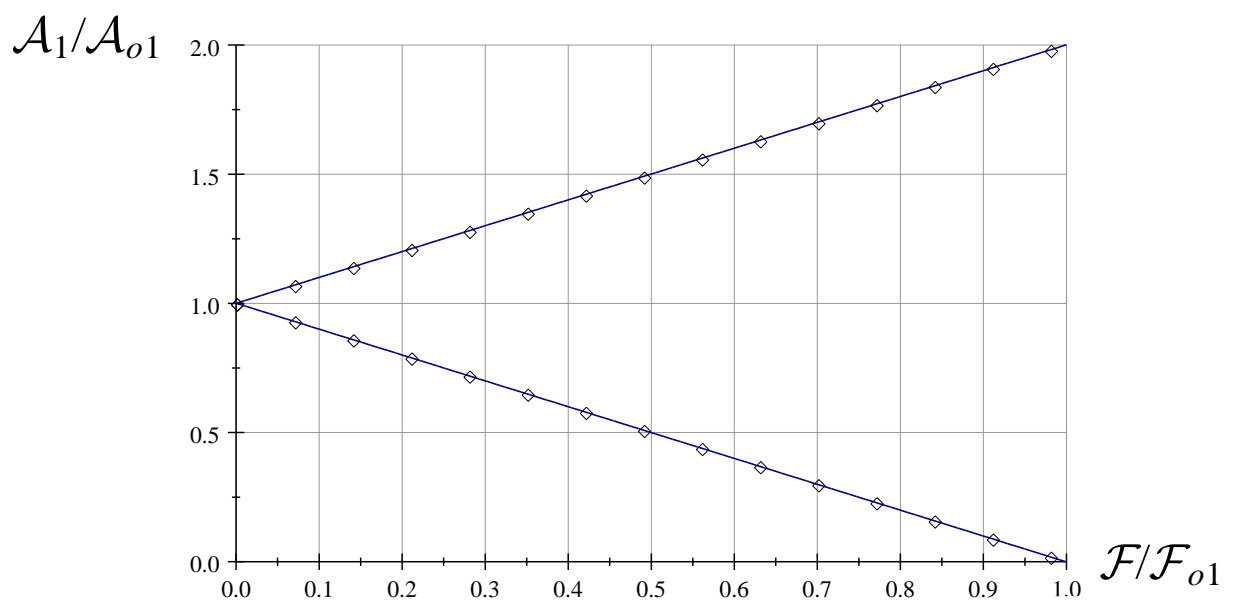

Figure 3 .

The approximate solutions obtained again by fitting a curve onto the computational results are practically linear functions. These are are also shown in Figure $3\left(\mathcal{A}_{o 1}=\right.$ $\left.24.838, \mathcal{F}_{o 1}=4.285\right)$. 


$$
\begin{aligned}
& \frac{\mathcal{A}_{1}}{\mathcal{A}_{o 1}}=1.0004-1.0007 \frac{\mathcal{F}}{\mathcal{F}_{o 1}} \simeq 1.000-1.000 \frac{\mathcal{F}}{\mathcal{F}_{o 1}} \\
& \frac{\mathcal{A}_{1}}{\mathcal{A}_{o 1}}=1.0004+1.0007 \frac{\mathcal{F}}{\mathcal{F}_{o 1}} \simeq 1.000+1.000 \frac{\mathcal{F}}{\mathcal{F}_{o 1}}
\end{aligned}
$$

The square of the first natural frequency of the plate when unloaded and the first critical load can be calculated by using equation (6.1a).

6.3. Spring supported plate. For a spring supported plate the results depend on the dimensionless spring constant $\mathcal{K}$ as well. If $i=1$ the table below shows the results we have obtained for $\mathcal{A}_{o 1}(\mathcal{K})$ and $\mathcal{F}_{o 1}(\mathcal{K})$.

TABLE 3

\begin{tabular}{|c|c|c|c|l|l|c|c|c|}
\hline $\mathcal{K}$ & 0.0 & 1.00 & 5.00 & 10.00 & 50.00 & 100.00 & 1000.00 & $\mathcal{K} \rightarrow \infty$ \\
\hline $\mathcal{A}_{o 1}$ & 24.838 & 37.36 & 63.118 & 76.677 & 96.791 & 100.41 & 103.97 & 104.85 \\
\hline $\mathcal{F}_{o 1}$ & 4.285 & 6.498 & 10.481 & 12.182 & 14.115 & 14.397 & 14.657 & 14.68 \\
\hline
\end{tabular}

Observe that for $\mathcal{K}=0$ and $\mathcal{K} \rightarrow \infty$ Table 3 contains the values valid for simply supported and clamped plates. For a compressive $f$ the following curves can be fitted onto the results obtained:

$$
\begin{array}{ll}
\mathcal{K}=1.00 & \frac{\mathcal{A}_{1}}{\mathcal{A}_{o 1}}=0.9937-1.0067 \frac{\mathcal{F}}{\mathcal{F}_{o 1}} \\
\mathcal{K}=5.00 & \frac{\mathcal{A}_{1}}{\mathcal{A}_{o 1}}=1.000-0.99119 \frac{\mathcal{F}}{\mathcal{F}_{o 1}}-8.7863 \times 10^{-3}\left(\frac{\mathcal{F}}{\mathcal{F}_{o 1}}\right)^{2} \\
\mathcal{K}=10.00 & \frac{\mathcal{A}_{1}}{\mathcal{A}_{o 1}}=1.000-0.98223 \frac{\mathcal{F}}{\mathcal{F}_{o 1}}-1.7763 \times 10^{-2}\left(\frac{\mathcal{F}}{\mathcal{F}_{o 1}}\right)^{2} \\
\mathcal{K}=50.00 & \frac{\mathcal{A}_{1}}{\mathcal{A}_{o 1}}=1.000-0.9721 \frac{\mathcal{F}}{\mathcal{F}_{o 1}}-2.7839 \times 10^{-2}\left(\frac{\mathcal{F}}{\mathcal{F}_{o 1}}\right)^{2} \\
\mathcal{K}=100.00 & \frac{\mathcal{A}_{1}}{\mathcal{A}_{o 1}}=1.000-0.9712 \frac{\mathcal{F}}{\mathcal{F}_{o 1}}-2.82 \times 10^{-2}\left(\frac{\mathcal{F}}{\mathcal{F}_{o 1}}\right)^{2} \\
\mathcal{K}=1000.00 & \frac{\mathcal{A}_{1}}{\mathcal{A}_{o 1}}=0.99997-0.97108 \frac{\mathcal{F}}{\mathcal{F}_{o 1}}-2.8827 \times 10^{-2}\left(\frac{\mathcal{F}}{\mathcal{F}_{o 1}}\right)^{2}
\end{array}
$$

For a tensile $f$ equations 6.5 are the polynomials we have fitted onto the computational results.

$$
\begin{array}{ll}
\mathcal{K}=1.00 & \frac{\mathcal{A}_{1}}{\mathcal{A}_{o 1}}=0.99364+1.0068 \frac{\mathcal{F}}{\mathcal{F}_{o 1}} \\
\mathcal{K}=5.00 & \frac{\mathcal{A}_{1}}{\mathcal{A}_{o 1}}=1.0002+0.99203 \frac{\mathcal{F}}{\mathcal{F}_{o 1}}-4.511 \times 10^{-3}\left(\frac{\mathcal{F}}{\mathcal{F}_{o 1}}\right)^{2} \\
\mathcal{K}=10.00 & \frac{\mathcal{A}_{1}}{\mathcal{A}_{o 1}}=1.0004+0.98359 \frac{\mathcal{F}}{\mathcal{F}_{o 1}}-8.6524 \times 10^{-3}\left(\frac{\mathcal{F}}{\mathcal{F}_{o 1}}\right)^{2}
\end{array}
$$




$$
\begin{array}{ll}
\mathcal{K}=50.00 & \frac{\mathcal{A}_{1}}{\mathcal{A}_{o 1}}=1.0006+0.97411 \frac{\mathcal{F}}{\mathcal{F}_{o 1}}-1.4003 \times 10^{-2}\left(\frac{\mathcal{F}}{\mathcal{F}_{o 1}}\right)^{2} \\
\mathcal{K}=100.00 & \frac{\mathcal{A}_{1}}{\mathcal{A}_{o 1}}=1.0006+0.97360 \frac{\mathcal{F}}{\mathcal{F}_{o 1}}-1.4546 \times 10^{-2}\left(\frac{\mathcal{F}}{\mathcal{F}_{o 1}}\right)^{2} \\
\mathcal{K}=1000.00 & \frac{\mathcal{A}_{1}}{\mathcal{A}_{o 1}}=1.0047+0.94510 \frac{\mathcal{F}}{\mathcal{F}_{o 1}}+1.8687 \times 10^{-2}\left(\frac{\mathcal{F}}{\mathcal{F}_{o 1}}\right)^{2}
\end{array}
$$

Observe that functions (6.4) and (6.5) are almost linear functions. For this reason Table 4 presents the computational results if $\mathcal{K}=100.0$ only.

TABLE 4

\begin{tabular}{|l|l|l|l|l|l|l|l|}
\hline $\mathcal{F} / \mathcal{F}_{o 1}$ & 0.069 & 0.139 & 0.208 & 0.278 & 0.347 & 0.417 & 0.486 \\
\hline $\mathcal{A} / \mathcal{A}_{o 1}$ - compression & 0.933 & 0.865 & 0.796 & 0.729 & 0.659 & 0.590 & 0.521 \\
$\mathcal{A} / \mathcal{A}_{o 1}-$ tension & 1.068 & 1.136 & 1.203 & 1.270 & 1.337 & 1.404 & 1.471 \\
\hline \hline $\mathcal{F} / \mathcal{F}_{o 1}$ & 0.556 & 0.625 & 0.695 & 0.764 & 0.834 & 0.903 & 0.972 \\
\hline $\mathcal{A} / \mathcal{A}_{o 1}-$ compression & 0.452 & 0.382 & 0.312 & 0.241 & 0.171 & 0.100 & 0.028 \\
$\mathcal{A} / \mathcal{A}_{o 1}-$ tension & 1.537 & 1.604 & 1.670 & 1.736 & 1.802 & 1.868 & 1.934 \\
\hline
\end{tabular}

Figure 4 shows function $(6.4 \mathrm{e})$ and $6.5 \mathrm{e}$ fitted onto the above results.

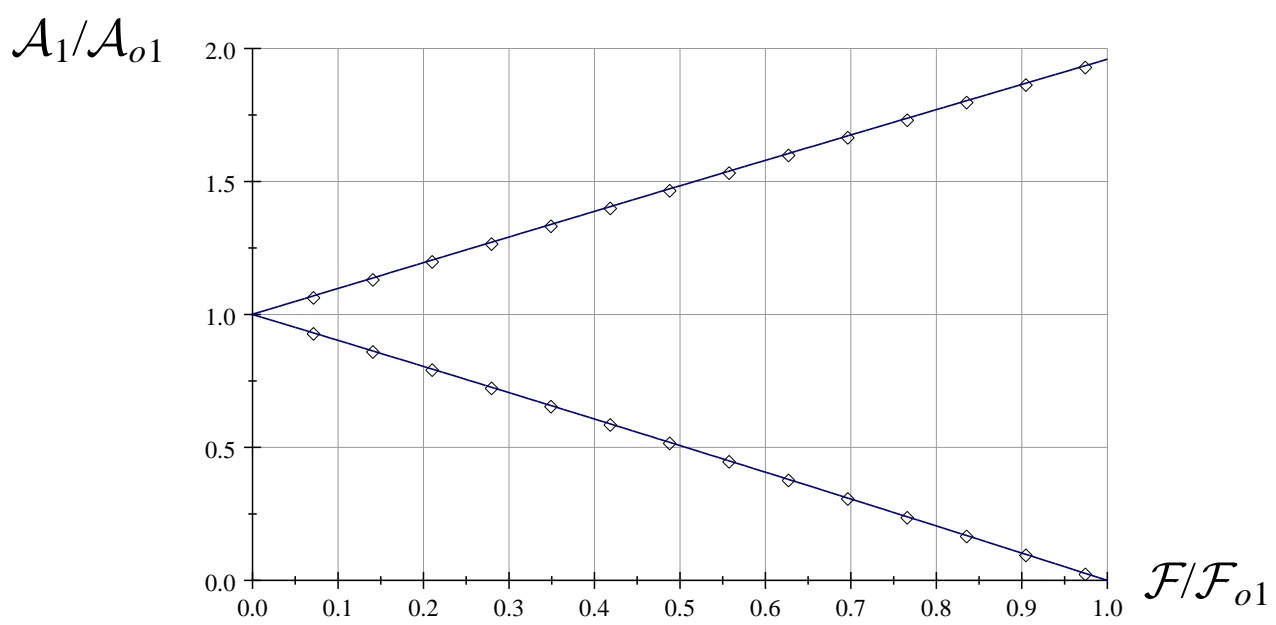

Figure 4 .

\section{Concluding Remarks}

We have dealt with the vibrations of circular plates subjected to a constant radial load in its plane on the outer boundary. The load can be either compressive or tensile. When solving the problem we have assumed that the deformations due to the load are also axisymmetric. 
(a) We have determined the Green functions for three support arrangements - a clamped plate, a simply supported plate and a plate supported by a torsional spring on its outer boundary - and for tensile and compressive in-plane loads as well. With the Green functions, the self adjoint eigenvalue problems giving the natural frequencies of the vibrations for the circular plates and loads considered have been replaced by six eigenvalue problems each of which is governed by a Fredholm integral equation.

(b) If the plate is subjected to a load perpendicular to the middle plane the deflections can be determined by integration - see equation 4.28.

(c) The eigenvalue problems governed by the Fredholm integral equations are reduced to algebraic eigenvalue problems and the eigenvalues as functions of the load are computed using the boundary element method and the QZ algorithm. According to the results the square of the first natural frequency can be approximated with good accuracy (or accurately for a simply supported plate) by linear functions of the load for the parameters (material constants and geometrical data) considered.

(d) Though in Section 6 we have presented the computational results for the first eigenvalues only, it seems probable on the basis of the computational results that equation

$$
\frac{\mathcal{A}_{i}}{\mathcal{A}_{o i}} \approx 1.000-1.000 \frac{\mathcal{F}}{\mathcal{F}_{o i}}
$$

provides a very good approximation if $i>1$.

Finally we remark that investigations for annular plates are in progress. Some preliminary results for compressive loads were presented earlier [18, 2005], [19, 2005].

\section{Appendix A. Bessel functions - FUndamental RELATions}

Let us denote $J_{n}(x)$ and $Y_{n}(x)$ uniformly by $H_{n}(x)$. The following relations hold

$$
\begin{aligned}
\frac{d H_{0}(x)}{d x} & =-H_{1}(x), \\
\frac{d H_{1}(x)}{d x} & =\frac{1}{x}\left(H_{0}(x) x-H_{1}(x)\right)=H_{0}(x)-\frac{1}{x} H_{1}(x)=\frac{1}{2}\left(H_{0}(x)-H_{2}(x)\right), \\
\frac{d H_{n}(x)}{d x} & =\frac{1}{2}\left(H_{n-1}(x)-H_{n+1}(x)\right), \\
\frac{2 n}{x} H_{n}(x) & =H_{n-1}(x)+H_{n+1}(x),
\end{aligned}
$$

and

See [20, 1977a] for more details.

$$
Y_{n}(x) J_{n+1}(x)-Y_{n+1}(x) J_{n}(x)=\frac{2}{\pi x} .
$$

As regards the Bessel functions $I_{n}(x)$ and $K_{n}(x)$, use has been made of the following relations

$$
\begin{aligned}
& \frac{d I_{0}(x)}{d x}=I_{1}(x), \quad \frac{d K_{0}(x)}{d x}=-K_{1}(x), \\
& \frac{d I_{1}(x)}{d x}=\frac{1}{x}\left(x I_{0}(x)-I_{1}(x)\right)=I_{0}(x)-\frac{1}{x} I_{1}(x)=\frac{1}{2}\left(I_{0}(x)+I_{2}(x)\right),
\end{aligned}
$$




$$
\begin{aligned}
\frac{d I_{n}(x)}{d x} & =\frac{1}{2}\left(I_{n-1}(x)+I_{n+1}(x)\right) \\
\frac{2 n}{x} I_{n}(x) & =I_{n-1}(x)-I_{n+1}(x) \\
\frac{d K_{1}(x)}{d x} & =-\frac{1}{x}\left(x K_{0}(x)+K_{1}(x)\right)=-K_{0}(x)-\frac{1}{x} K_{1}(x)=-\frac{1}{2}\left(K_{0}(x)+K_{2}(x)\right) \\
\frac{d K_{n}(x)}{d x} & =-\frac{1}{2}\left(K_{n-1}(x)+K_{n+1}(x)\right) \\
-\frac{2 n}{x} I_{n}(x) & =K_{n-1}(x)-K_{n+1}(x)
\end{aligned}
$$

and

$$
I_{n}(x) K_{n+1}(x)+I_{n+1}(x) K_{n}(x)=\frac{1}{x} .
$$

See [20, 1977b] for more details.

Appendix B. Clamped plate - Calculation of the integration constants

After solving equation system 4.10 we have

$$
\begin{aligned}
& A_{1}=\frac{1}{2 \pi f}\left(\ln \xi-\frac{J_{01}-J_{0 \xi}}{\sqrt{\mathcal{F}} J_{11}}\right), \quad A_{3}=\frac{1}{4 f}\left(\frac{2}{\pi \sqrt{\mathcal{F}} J_{11}}-Y_{0 \xi}+\frac{Y_{11} J_{0 \xi}}{J_{11}}\right), \\
& B_{1}=\frac{1}{2 \pi f} \frac{J_{0 \xi}-J_{01}}{\sqrt{\mathcal{F}} J_{11}}, \quad B_{2}=\frac{1}{2 \pi f}, \quad B_{3}=\frac{1}{2 \pi f}\left(\frac{1}{\sqrt{\mathcal{F}} J_{11}}+\frac{\pi Y_{11} J_{0 \xi}}{2 J_{11}}\right)
\end{aligned}
$$

and

$$
B_{4}=-\frac{1}{2 f \pi} \frac{\pi J_{0 \xi}}{2} .
$$

Substitution of the solutions above into 4.2 yields the Green function for the clamped plate.

\section{REFERENCES}

1. R. Lawther: On the straightness of eigenvalue iterations. Computational Mechanics, 37, (2006), 362-368.

2. S. Syngellakis and A. Elzein: Plate buckling loads by the the boundary element method. International Journal for Numerical Methods in Engineering, 37, (1994), 17631778 .

3. M. S. NeRANTZAKI and J. T. KATsiKadelis: Buckling of plates with variable thickness - an analog solution. Engineering Analysis with Boundary elements, 18, (1996), 149-154.

4. J. Lin, R. C. Duffield and H. Shiн: Buckling analysis of elastic plates by boundary element method. Engineering Analysis with Boundary Elements, 23, (1999), 131-137.

5. P. H. Wen, M. H. Aliabadi and A. Young: Application of dual reciprocity method to plates and shells. Engineering Analysis with Boundary elements, 24, (2000), 583-590.

6. J. Purbolaksono and M. H. Aliabadi: Buckling analysis of shear deformable plates by boundary element method. International Journal for Numerical Methods in Engineering, 62, (2005), 537-563. 
7. C.D. Mote JR: Free vibrations of initially stressed circular disks. Journal of Engineering for Industry, 87, (1965), 258-364.

8. G. C. Pardoen: Vibration and buckling analysis of axisymmetric polar orthotropic circular plates. Computers \&5 Structures, 4, (1974), 951-960.

9. D. Снотоva: Vibrations of circular plates subjected to an in plane load. Master thesis (in Hungarian), Department of Mecahnics, University of Miskolc, Hungary, 1980.

10. L. W. Chen and J. L. Dong: Large amplitude vibration of an initially stressed hick circular plate. AIAA, 21, (1983), 1317-1324.

11. L. W. Chen and J. L. Dong: Vibrations of an initially stressed transversely isotropic circular thick plate. International Journal of Mechanical Sciences, 26(4), (1984), 253263.

12. A. Tylikowskia and K. Frischmuthi: Stability and stabilization of circular plate parametric vibrations. International Journal of Solids and Structures, 40, (2003), 51875196.

13. D. Younesian and M. H. Aleghafourian: A direct formulation and numerical solution of the general transient elastodynamic problem. International Journal of Civil Engineering and Building Materials, 2(4), (2012), 167-174.

14. N. SzÜCS: Vibrations of circular plates subjected to an in-plane load. GÉP, LVIII(5-6), (2007), 41-47. (in Hungarian).

15. P. ZdeněK Bažant and L. Cedolin: Stability of structures, Elastic, Inelastic, Fracture, and Damage Theories (2nd edition). Dover Publications, Inc. Mineola, New York, 2003, p. 427.

16. I. KozÁK: Strength of materials V. - Thin walled structures and the theory of plates and shells. Tankönyvkiadó (Publisher of Textbooks), Budapest, Hungary, 1967, pp. 287-291. (in Hungarian).

17. C. T. H. BAKER: The Numerical Treatment of Integral Equations - Monographs on Numerical Analysis. Clarendon Press, Oxford, 1977.

18. G. Szeidl, D. Georgieva and N. SzüCS: Vibration of a circular plate with a hole subjected to a radial uniform load in its plane. In Section G: Applied Mechanics, Modern Numerical Methods, pages 149-154, microCAD 2005, University of Miskolc, March 10-11, 2005.

19. G. SzeidL, N. SzüCS and B. Tóth: Vibration of circular plates subjected to uniform loads in their plane. In V. Kompis, editor, Numerical Methods on Continuum Mechanics and 4th Workshop on Trefftz Methods, Slovakia, August 23-26, 2005., Extended six page abstract on the conference CD.

20. E. Janke, F. Emde and F. Lösch: Tafelen Höheren Funktionen. Nauka, Moscow, 1977, (a) p. 241-242 and (b) 245-246. (Russian edition.) 